\title{
${ }^{18}$ F-Alfatide II PET/CT for Identification of Breast Cancer: A Preliminary Clinical Study
}

\author{
Jiang $\mathrm{Wu}^{* 1}$, Shaohua Wang*2, Xianzhong Zhang ${ }^{3}$, Zhaogang Teng ${ }^{4}$, Jingjie Wang ${ }^{2}$, Bryant C. Yung ${ }^{5}$, Gang Niu ${ }^{5}$, \\ Hong $\mathrm{Zhu}^{1}$, Guangming $\mathrm{Lu}^{4}$, and Xiaoyuan $\mathrm{Chen}^{5}$ \\ ${ }^{I}$ Department of Nuclear Medicine, Jinling Hospital, School of Medicine, Nanjing University, Nanjing, China; ${ }^{2}$ Department of General \\ Surgery, Jinling Hospital, School of Medicine, Nanjing University, Nanjing, China; ${ }^{3}$ Center for Molecular Imaging and Translational \\ Medicine, Xiamen University, Xiamen, China; ${ }^{4}$ Department of Medical Imaging, Jinling Hospital, School of Medicine, Nanjing \\ University, Nanjing, China; and ${ }^{5}$ Laboratory of Molecular Imaging and Nanomedicine, National Institute of Biomedical Imaging and \\ Bioengineering, National Institutes of Health, Bethesda, Maryland
}

\begin{abstract}
${ }^{18} \mathrm{~F}$-alfatide II has been proven to have excellent clinical translational potential. In this study, we investigated ${ }^{18} \mathrm{~F}$-alfatide II for identifying breast cancer and compared the performances between ${ }^{18} \mathrm{~F}$-alfatide II and ${ }^{18} \mathrm{~F}-\mathrm{FDG}$. Methods: Forty-four female patients with suspected primary breast cancer were recruited. PET/CT images using ${ }^{18} \mathrm{~F}$-alfatide II and ${ }^{18} \mathrm{~F}$-FDG were acquired within $7 \mathrm{~d}$. Tracer uptake in breast lesions was evaluated by visual analysis, and semiquanti-

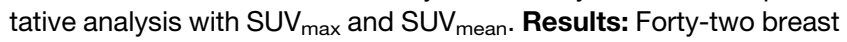
cancer lesions and 11 benign breast lesions were confirmed by histopathology in 44 patients. Both ${ }^{18} \mathrm{~F}$-alfatide II and ${ }^{18} \mathrm{~F}$-FDG had higher uptake in breast cancer lesions than in benign breast lesions ( $P<0.05$ for ${ }^{18} \mathrm{~F}$-alfatide II, $P<0.05$ for $\left.{ }^{18} \mathrm{~F}-\mathrm{FDG}\right)$. The area under the curve of ${ }^{18} \mathrm{~F}$-alfatide II was slightly less than that of ${ }^{18} \mathrm{~F}$ FDG. Both ${ }^{18} \mathrm{~F}$-alfatide II and ${ }^{18} \mathrm{~F}-\mathrm{FDG}$ had high sensitivity $(88.1 \%$ vs. $90.5 \%)$, high positive predictive value ( $88.1 \%$ vs. $88.4 \%)$, moderate specificity (54.5\% vs. $54.5 \%$ ), and moderate negative predictive value (54.5\% vs. $60.0 \%$ ) for differentiating breast cancer from benign breast lesions. By combining ${ }^{18} \mathrm{~F}$-alfatide II and ${ }^{18} \mathrm{~F}-\mathrm{FDG}$, the sensitivity and negative predictive value significantly increased to $97.6 \%$ and $85.7 \%$, respectively, with positive predictive value slightly increased to $89.1 \%$ and no change to the specificity (54.5\%). The uptake of ${ }^{18} \mathrm{~F}$-alfatide II $\left(\mathrm{SUV}_{\text {max }}: 3.77 \pm 1.78\right)$ was significantly lower than that of ${ }^{18} \mathrm{~F}-\mathrm{FDG}$ $\left(\mathrm{SUV}_{\max }: 7.37 \pm 4.48\right)$ in breast cancer lesions $(P<0.05) .{ }^{18} \mathrm{~F}$-alfatide II uptake in triple-negative subtype was significantly lower than that in luminal A and luminal B subtypes. By contrast, human epidermal growth factor receptor-2 (HER-2)-overexpressing subtype had higher ${ }^{18} \mathrm{~F}-\mathrm{FDG}$ uptake than the other 3 subtypes. There were 8 breast cancer lesions with higher ${ }^{18} \mathrm{~F}$-alfatide II uptake than ${ }^{18} \mathrm{~F}$-FDG uptake, which all had a common characteristic that HER-2 expression was negative and estrogen receptor expression was strongly positive. Conclusion: ${ }^{18} \mathrm{~F}$-alfatide II is suitable for clinical use in breast cancer patients. ${ }^{18} \mathrm{~F}$-alfatide II is of good performance, but not superior to ${ }^{18} \mathrm{~F}-\mathrm{FDG}$ in identifying breast cancer. ${ }^{18} \mathrm{~F}$-alfatide II may have superiority to ${ }^{18} \mathrm{~F}-\mathrm{FDG}$ in detecting breast cancer with strongly positive estrogen receptor expression and negative HER-2 expression.
\end{abstract}

Received Jan. 19, 2018; revision accepted Apr. 16, 2018.

For correspondence or reprints contact either of the following:

Xiaoyuan Chen, Laboratory of Molecular Imaging and Nanomedicine,

National Institute of Biomedical Imaging and Bioengineering, National

Institutes of Health, 31 Center Dr., 1C22, Bethesda, MD 20892.

E-mail: shawn.chen@nih.gov

Guangming Lu, Department of Medical Imaging, Jinling Hospital, School of Medicine, Nanjing University, 305 East Zhongshan Rd., Nanjing, China 210002.

E-mail: cjr.luguangming@vip.163.com

${ }^{*}$ Contributed equally to this work.

Published online Apr. 26, 2018.

COPYRIGHT (C 2018 by the Society of Nuclear Medicine and Molecular Imaging.
Key Words: ${ }^{18} \mathrm{~F}$-alfatide II; integrin $\mathrm{a}_{\mathrm{v}} \beta_{3}$; positron emission tomography; breast cancer

J Nucl Med 2018; 59:1809-1816

DOI: 10.2967/jnumed.118.208637

\section{A}

ngiogenesis exerts a prominent role in promoting tumor growth, progression, and metastasis. Integrin $\alpha_{v} \beta_{3}$ is highly expressed on activated endothelial cells of tumor neovasculature and thus is key to tumor angiogenesis (1-3). Arginine-glycineaspartate (RGD) peptides have a high binding affinity with integrin $\alpha_{v} \beta_{3}$. As a result, a variety of RGD-based molecular probes have been developed to visualize integrin $\alpha_{\mathrm{v}} \beta_{3}$ expression (4-6). Because of the superiority of PET molecular imaging technique, RGD tracers labeled with positron-emitting radionuclides such as ${ }^{18} \mathrm{~F},{ }^{64} \mathrm{Cu},{ }^{68} \mathrm{Ga}$, and ${ }^{89} \mathrm{Zr}$ have attracted much attention $(7-10)$. As a PET tracer based on dimeric RGD peptide, ${ }^{18} \mathrm{~F}$-alfatide II has been recently proven to possess excellent clinical translational potential in several studies (11-16). Consequently, it warrants further promotion in clinical applications.

Breast cancer is a heterogeneous disease, and angiogenesis is one of the important characteristics (17). Great strides have been made in breast cancer treatment, such as endocrine therapy, targeted human epidermal growth factor receptor-2 (HER-2) therapy, and antiangiogenic therapy. Bevacizumab, as an antiangiogenic drug, was approved by the U.S. Food and Drug Administration to treat patients with advanced breast cancer in 2008. Three years later, however, this approval was withdrawn due to a lack of evidence in improving overall survival. The unsuccessful predicament of bevacizumab could be attributed to the absence of prescreening to select for patients with specific angiogenic biomarkers (18). Accordingly, imaging angiogenesis is crucial for breast cancer patients before antiangiogenic therapy. Moreover, the present molecular classification of breast cancer is based on the status of estrogen receptor (ER), progesterone receptor (PR), and HER-2, which are all expressed on the membrane of breast cancer cells themselves. The biomarkers located in breast cancer stroma are not included in the present classification. With continuous development and improvement of the molecular classification system, some stromal biomarkers such as integrin $\alpha_{v} \beta_{3}$ might be incorporated into new classifications in the future. Consequently, the molecular 


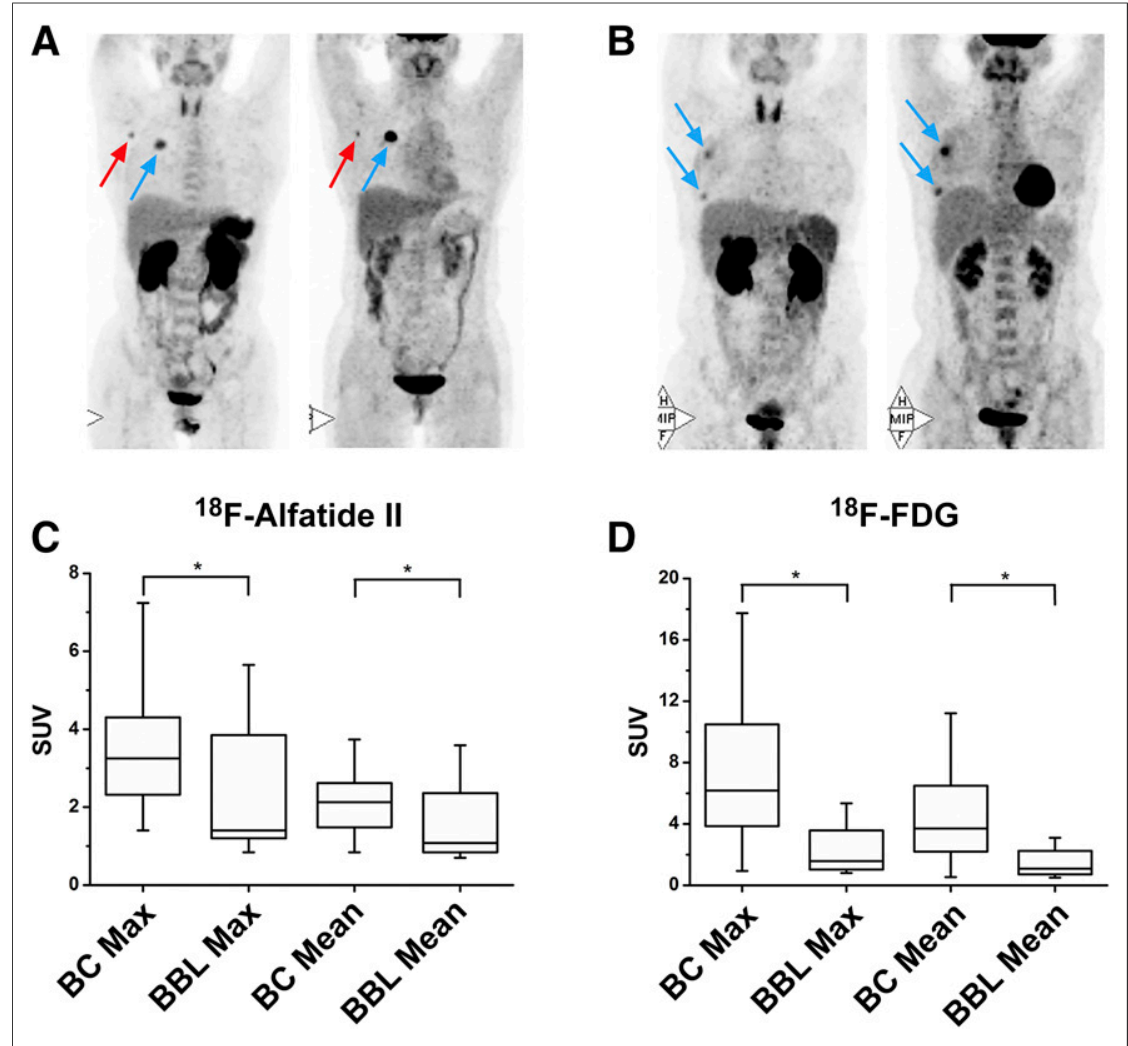

FIGURE 1. (A) A 50-y-old patient with HER-2-overexpressing breast cancer (blue arrow), and axillary lymph node metastasis (red arrow) showing high ${ }^{18} \mathrm{~F}$-alfatide II and ${ }^{18} \mathrm{~F}$-FDG uptake. (B) A 47-y-old patient with 2 lesions of breast intraduct papilloma (blue arrows) showing high ${ }^{18} \mathrm{~F}$-alfatide II and ${ }^{18} \mathrm{~F}$-FDG uptake. Difference of SUVs between breast cancer and benign breast lesion for ${ }^{18} \mathrm{~F}$-alfatide II (C) and ${ }^{18} \mathrm{~F}$-FDG (D). ${ }^{*} P<0.05$.

imaging visualization of integrin $\alpha_{v} \beta_{3}$ expression should be valuable in exploring the molecular classification based on breast cancer stroma.

There have been a few studies using RGD-based PET probes such as ${ }^{18} \mathrm{~F}$-galacto-RGD, ${ }^{18} \mathrm{~F}-\mathrm{AH} 111585,{ }^{18} \mathrm{~F}-\mathrm{FPPRGD} 2$, and ${ }^{68} \mathrm{Ga}-\mathrm{PRGD} 2$ in the clinic for breast cancer patients (19-24). Generally, these PET tracers have shown satisfactory performance in terms of safety, feasibility, and usefulness. However, the numbers of breast cancer cases included in these studies are often very small. Some diagnostic parameters such as sensitivity, specificity, positive predictive value (PPV), and negative predictive value (NPV) are unknown for RGD-based PET tracers due to the absence of benign breast lesions in these studies. Moreover, ductal carcinoma in situ (DCIS) cases were not included in these investigations. For this reason, the information concerning RGD uptake in early breast cancer patients remains unclear. Furthermore, there have been inadequate studies regarding RGD uptake by different subtypes based on the present molecular classification of breast cancer.

In this work, ${ }^{18} \mathrm{~F}$-alfatide II was investigated in clinically suspected breast cancer patients for the first time, and it was meanwhile compared with ${ }^{18} \mathrm{~F}-\mathrm{FDG}$. We obtained diagnostic parameters of ${ }^{18} \mathrm{~F}$-alfatide II facilitating the differentiation between breast cancer and benign breast lesions and further assessed its diagnostic performance in comparison with ${ }^{18} \mathrm{~F}$-FDG. We also investigated ${ }^{18} \mathrm{~F}$-alfatide II uptake in different molecular subtypes of breast cancer and evaluated the differences as compared with ${ }^{18} \mathrm{~F}$-FDG.

\section{MATERIALS AND METHODS}

\section{Patients}

This study was approved by the ethics committee of Jinling Hospital (approval no. 2015NZYW-007) and registered at ClinicalTrials.gov (NCT02582801). Inclusion criteria consisted of clinically suspected primary breast cancer according to conventional imaging (e.g., mammography, ultrasound, MRI), no prior treatment for breast lesions, $18 \mathrm{y}<$ age $<70$ y. Exclusion criteria consisted of pregnancy, lactation period, and some accompanied serious diseases (e.g., impaired liver or renal function, active tuberculosis, other malignant tumor). Forty-four female patients (age range, 28-66 y; mean age $\pm \mathrm{SD}, 50.73 \pm 8.01$ y) were included in this study and each patient signed a written informed consent form. This was a preliminary clinical study about the diagnosis of breast cancer, and a study about treatment response monitoring is ongoing in a larger group of patients.

\section{PET/CT Acquisition and Image Analysis}

${ }^{18} \mathrm{~F}$-alfatide II was prepared according to the previously reported method (25). The injected activity was $306 \pm 80 \mathrm{MBq}$ (range, 155$503 \mathrm{MBq}$ ). No specific patient preparation such as fasting was requested for ${ }^{18} \mathrm{~F}$-alfatide II PET/CT scanning, which was performed at $60 \mathrm{~min}$ after the injection using a Biography $16 \mathrm{PET} / \mathrm{CT}$ scanner (Siemens Healthcare). CT acquisition was initially performed with $120 \mathrm{kV}, 140 \mathrm{~mA}$, and a slice thickness of $5 \mathrm{~mm}$. Immediately after $\mathrm{CT}$ acquisition, PET emission scanning was performed with an acquisition time of $3 \mathrm{~min}$ for each bed. PET data were corrected for attenuation using the coregistered CT data and PET images and reconstructed using

TABLE 1

Comparisons of ${ }^{18} \mathrm{~F}$-Alfatide II and ${ }^{18} \mathrm{~F}$-FDG Uptake Between Breast Cancer and Benign Breast Lesion

\begin{tabular}{|c|c|c|c|c|c|}
\hline \multirow[b]{2}{*}{ Disease } & & \multicolumn{2}{|c|}{${ }^{18} \mathrm{~F}$-alfatide II } & \multicolumn{2}{|c|}{${ }^{18} \mathrm{~F}-\mathrm{FDG}$} \\
\hline & & $\mathrm{SUV}_{\max }$ & $S U V_{\text {mean }}$ & $\mathrm{SUV}_{\max }$ & $S U V_{\text {mean }}$ \\
\hline \multirow[t]{2}{*}{$\mathrm{BC}$} & & $3.77 \pm 1.78$ & $2.25 \pm 0.98$ & $7.37 \pm 4.48$ & $4.54 \pm 2.82$ \\
\hline & $P$ value & $P<0.05$ & $P<0.05$ & $P<0.05$ & $P<0.05$ \\
\hline BBL & & $2.37 \pm 1.62$ & $1.50 \pm 0.92$ & $2.88 \pm 2.77$ & $1.75 \pm 1.50$ \\
\hline
\end{tabular}




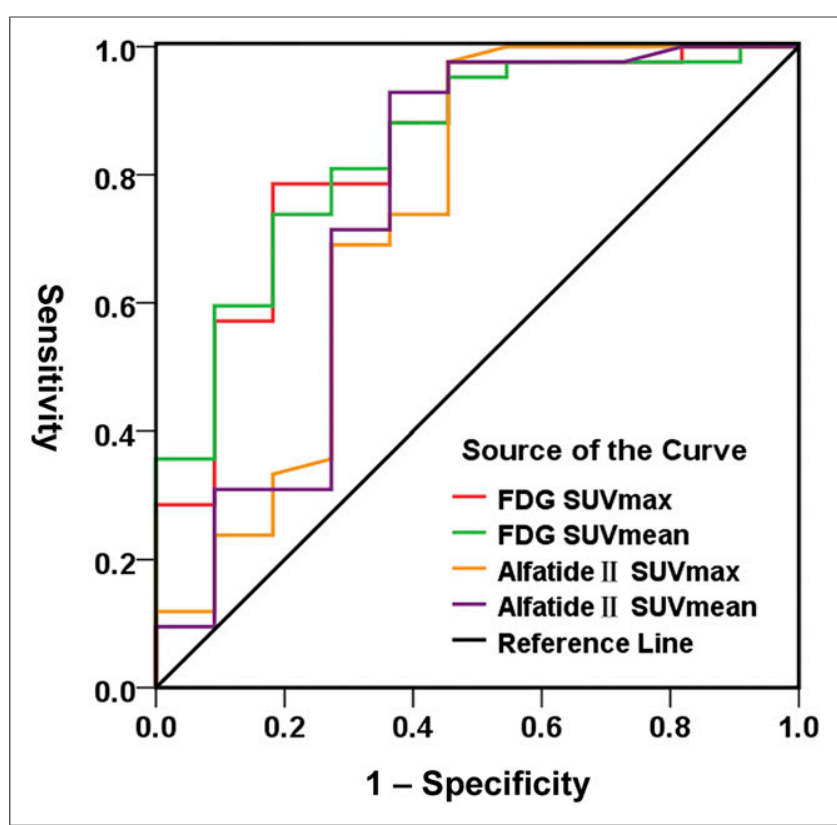

FIGURE 2. ROC curves of SUVs of ${ }^{18} \mathrm{~F}$-alfatide II and ${ }^{18} \mathrm{~F}-\mathrm{FDG}$ in differentiating breast cancer from benign lesion.

an iterative algorithm. PET, CT, and fused images were displayed on a Siemens/Syngo user interface.

${ }^{18} \mathrm{~F}-\mathrm{FDG}$ PET/CT was performed within $1-7 \mathrm{~d}$ before or after ${ }^{18} \mathrm{~F}-$ alfatide II PET/CT. All patients fasted for at least $6 \mathrm{~h}$ before receiving an intravenous injection of ${ }^{18} \mathrm{~F}-\mathrm{FDG}(\sim 3.7 \mathrm{MBq} / \mathrm{kg}$ of body weight). Blood glucose was measured before injection to ensure that the level was below $140 \mathrm{mg} / \mathrm{dL}$. The same procedure was used for ${ }^{18} \mathrm{~F}-\mathrm{FDG}$ $\mathrm{PET} / \mathrm{CT}$ data acquisition as was used for ${ }^{18} \mathrm{~F}$-alfatide II PET/CT using the same scanner. The uptake time between ${ }^{18} \mathrm{~F}-\mathrm{FDG}$ injection and $\mathrm{PET} / \mathrm{CT}$ acquisition was also $60 \mathrm{~min}$.

The images from 2 PET/CT scans were visually interpreted by a consensus of 2 experienced nuclear medicine physicians, who were masked to the histologic diagnosis and other imaging results. The $\mathrm{SUV}_{\text {max }}$ and the $\mathrm{SUV}_{\text {mean }}$ were semiquantitatively measured by drawing regions of interest over the breast lesions.

\section{Statistical Analysis}

The data were presented as the mean $\pm \mathrm{SD}$. The difference between 2 groups was analyzed by Student $t$ test, and differences among 3 or more groups were determined by ANOVA. Receiver-operating-characteristic (ROC) curve analysis was performed to evaluate the diagnostic performance. The area under the curve (AUC) and the cutoff value were further determined at the point with the highest Youden index. The sensitivity, specificity, PPV, and NPV were calculated to compare the differences in diagnostic accuracy. A Pearson correlation coefficient test was performed to determine the correlation between ${ }^{18} \mathrm{~F}$-alfatide II and ${ }^{18} \mathrm{~F}$-FDG groups. Statistical analysis was performed using SPSS software (version 17.0; SPSS, Inc.), and a $P$ value of less than 0.05 was considered statistically significant.

\section{RESULTS}

Performance of ${ }^{18} \mathrm{~F}$-Alfatide II in Diagnosis of Breast Cancer

All patients received biopsy or surgery after undergoing 2 PET/ CT scans. Fifty-three breast lesions were confirmed by histopathology in 44 patients. Among them, 42 lesions were malignant and the others were benign. The malignant lesions included DCIS (4 cases) and invasive carcinoma (38 cases). The latter was divided into luminal A subtype (6 cases), luminal B subtype (15 cases), HER-2-overexpressing subtype (5 cases), and triple-negative subtype (12 cases). In luminal B subtype, 6 cases were HER-2-positive and 9 cases were HER-2 -negative. Luminal B subtype with positive HER-2 expression is completely different from HER-2-overexpressing subtype (non-luminal subtype). The former is defined as ER- and (or) PR-positive, HER-2-positive, and Ki-67 at any level. The latter is defined as both ER- and PRnegative and HER-2-positive. The benign lesions were composed of breast fibroadenoma (4 cases), breast adenosis (4 cases), breast intraduct papilloma ( 2 cases), and mastitis (1 case).

Most breast cancer lesions had high uptake (Fig. 1A). Several benign breast lesions also showed increased uptake (Fig. 1B). The $\mathrm{SUV}_{\text {max }}$ and $\mathrm{SUV}_{\text {mean }}$ of breast lesions are shown in Table 1. In general, breast cancer lesions had higher ${ }^{18} \mathrm{~F}$-alfatide II uptake than benign breast lesions $(P<0.05)$. Also, ${ }^{18} \mathrm{~F}-\mathrm{FDG}$ accumulation in breast cancer lesions was more intense than that in benign breast lesions $(P<0.05)$. However, the difference of ${ }^{18} \mathrm{~F}$-alfatide II SUV between breast cancer and benign lesions was less significant as compared with that of ${ }^{18}$ F-FDG SUV (Figs. 1C and 1D).

As shown in Figure 2, ROC curves of ${ }^{18} \mathrm{~F}$-alfatide II are located at the upper left of the chance line, just as those of ${ }^{18} \mathrm{~F}-\mathrm{FDG}$, indicating their strong potential for identifying breast cancer. However, the AUC of ${ }^{18} \mathrm{~F}$-alfatide II is slightly less than that of ${ }^{18} \mathrm{~F}-\mathrm{FDG}$. The corresponding statistics of the ROC curves are shown in Table 2 . The maximum Youden index of ${ }^{18} \mathrm{~F}$-alfatide II $\mathrm{SUV}_{\max }(52.1 \%)$ is lower than that of ${ }^{18} \mathrm{~F}-\mathrm{FDG} \mathrm{SUV}_{\max }$ $(60.4 \%)$, but the gap is small. Moreover, the indices are very close between ${ }^{18} \mathrm{~F}$-alfatide II $\mathrm{SUV}_{\text {mean }}(56.5 \%)$ and ${ }^{18} \mathrm{~F}$-FDG $\mathrm{SUV}_{\text {mean }}$ $(55.6 \%)$. The cutoff value with a maximum Youden index is 1.6 for ${ }^{18} \mathrm{~F}$-alfatide II SUV $\max , 1.28$ for ${ }^{18} \mathrm{~F}$-alfatide II SUV $\mathrm{SUan}_{\text {mean }}, 3.68$ for ${ }^{18} \mathrm{~F}-\mathrm{FDG} \mathrm{SUV}_{\max }$, and 2.26 for ${ }^{18} \mathrm{~F}-\mathrm{FDG} \mathrm{SUV}_{\text {mean. }}$. When the $\mathrm{SUV}_{\text {max }}$ or $\mathrm{SUV}_{\text {mean }}$ of the breast lesion is higher than its cutoff value, breast cancer may be considered. However, ${ }^{18} \mathrm{~F}$-FDG SUVmean has the largest AUC (0.84) and ${ }^{18} \mathrm{~F}$-alfatide II $\mathrm{SUV}_{\max }$ has the smallest AUC (0.738).

The visual analysis showed that both ${ }^{18} \mathrm{~F}$-alfatide II and ${ }^{18} \mathrm{~F}$ FDG for differentiating breast cancer from benign breast lesions had high sensitivity $(88.1 \%$ vs. $90.5 \%)$ and PPV $(88.1 \%$ vs. $88.4 \%)$. However, they had the same specificity $(54.5 \%)$ and

TABLE 2

ROC Quantitative Analysis of ${ }^{18} \mathrm{~F}$-Alfatide II and ${ }^{18} \mathrm{~F}-\mathrm{FDG}$ in Differentiating Breast Cancer from Benign Breast Lesion

\begin{tabular}{cccccc}
\hline Tracer & Cutoff value & AUC & Sensitivity & Specificity & Youden index \\
\hline${ }^{18}$ F-alfatide II & SUV $_{\max } 1.6$ & 0.738 & $97.6 \%$ & $54.5 \%$ & $52.1 \%$ \\
& SUV $_{\operatorname{mean}} 1.28$ & 0.752 & $92.9 \%$ & $63.6 \%$ & $56.5 \%$ \\
\multirow{2}{*}{${ }^{18}$ F-FDG } & SUV $_{\max } 3.68$ & 0.838 & $78.6 \%$ & $81.8 \%$ & $60.4 \%$ \\
& SUV $_{\text {mean }} 2.26$ & 0.840 & $73.8 \%$ & $81.8 \%$ & $55.6 \%$ \\
\hline
\end{tabular}




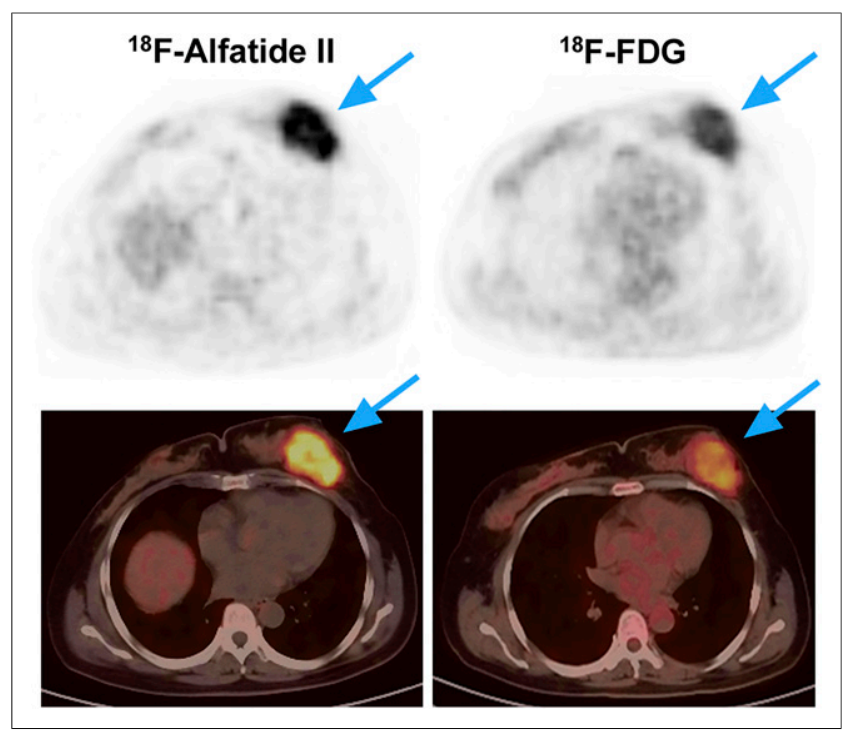

FIGURE 3. A 50-y-old patient with breast fibroadenoma (blue arrows) showing false-positive uptake of both ${ }^{18} \mathrm{~F}$-alfatide II (SUV $\left.\max : 5.65\right)$ and ${ }^{18}$ F-FDG (SUV $\left.\max : 3.57\right)$.

similar NPV ( $54.5 \%$ vs. $60 \%$ ), which were moderate. The falsepositive lesions were the same for ${ }^{18} \mathrm{~F}$-alfatide II and ${ }^{18} \mathrm{~F}-\mathrm{FDG}$, including 2 cases of breast fibroadenoma (Fig. 3), 1 case of breast adenosis, 1 case of breast intraduct papilloma, and 1 case of mastitis. One case of DCIS was false-negative for both ${ }^{18} \mathrm{~F}$-alfatide II and ${ }^{18} \mathrm{~F}$-FDG. Other false-negative lesions of ${ }^{18} \mathrm{~F}$-alfatide II included 4 cases of breast cancer with triple-negative subtype (Fig. 4). Two cases of breast cancer with luminal B subtype (HER-2-negative) and 1 case of invasive lobular carcinoma was also false-negative for ${ }^{18} \mathrm{~F}-\mathrm{FDG}$. As a result, the sensitivity and NPV significantly increased to $97.6 \%$ and $85.7 \%$, respectively, by combining ${ }^{18} \mathrm{~F}$-alfatide II and ${ }^{18} \mathrm{~F}$-FDG. However, there was no change on the specificity and only a slight increase on PPV (Table 3).
TABLE 3

Visual Analysis of ${ }^{18} \mathrm{~F}$-Alfatide II and ${ }^{18} \mathrm{~F}-\mathrm{FDG}$ in Differentiating Breast Cancer from Benign Breast Lesion

\begin{tabular}{lcccc}
\hline \multicolumn{1}{c}{ Tracer } & Sensitivity & Specificity & PPV & NPV \\
\hline${ }^{18}$ F-alfatide II & $88.1 \%$ & $54.5 \%$ & $88.1 \%$ & $54.5 \%$ \\
${ }^{18} \mathrm{~F}-$ FDG & $90.5 \%$ & $54.5 \%$ & $88.4 \%$ & $60.0 \%$ \\
$\begin{array}{c}18 \\
\text { F-alfatide II }+ \\
{ }^{18} \mathrm{~F}-\text { FDG }\end{array}$ & $97.6 \%$ & $54.5 \%$ & $89.1 \%$ & $85.7 \%$ \\
\hline
\end{tabular}

\section{Comparisons Between ${ }^{18} \mathrm{~F}$-Alfatide II and ${ }^{18} \mathrm{~F}$-FDG Uptake in Breast Cancer and Benign Breast Lesions}

The ${ }^{18} \mathrm{~F}$-alfatide II and ${ }^{18} \mathrm{~F}$-FDG uptake values in breast cancer and benign breast lesions are listed in Table 4 . The uptake of ${ }^{18} \mathrm{~F}$ alfatide II was significantly lower than that of ${ }^{18} \mathrm{~F}$-FDG in breast cancer lesions $(P<0.05)$, for both $\mathrm{SUV}_{\max }$ and $\mathrm{SUV}_{\text {mean. }}{ }^{18} \mathrm{~F}-$ alfatide II uptake in benign breast lesions was also lower than ${ }^{18} \mathrm{~F}$ FDG, but the difference was not statistically significant $(P>$ $0.05)$. The correlation between ${ }^{18} \mathrm{~F}$-alfatide II and ${ }^{18} \mathrm{~F}$-FDG uptake in breast cancer lesions was not significant $(r=0.03, P>0.05$ for $\mathrm{SUV}_{\text {max }}$ and $r=0.003, P>0.05$ for $\left.\mathrm{SUV}_{\text {mean }}\right)$. There was a positive correlation between ${ }^{18} \mathrm{~F}$-alfatide II and ${ }^{18} \mathrm{~F}$-FDG uptake in benign breast lesions, which was present for $\mathrm{SUV}_{\text {max }}(r=0.69$, $P<0.05)$ but absent for $\operatorname{SUV}_{\text {mean }}(r=0.59, P>0.05)$ (Fig. 5).

\section{${ }^{18}$ F-Alfatide II Uptake in Different Molecular Subtypes of Breast Cancer}

The ${ }^{18} \mathrm{~F}$-alfatide II and ${ }^{18} \mathrm{~F}-\mathrm{FDG}$ uptake values in different molecular subtypes of breast cancer are shown in Table 5. Luminal A, luminal $\mathrm{B}$, and HER-2-overexpressing subtypes have similar ${ }^{18} \mathrm{~F}$-alfatide II uptake values, with all being higher than those of triple-negative subtype. According to statistical analysis, there was significant difference in ${ }^{18} \mathrm{~F}$-alfatide II uptake between triple-negative subtype and luminal A subtype, as well as between triple-negative subtype and luminal B subtype $(P<0.05)$. No other 2 subtypes of breast cancer had significant difference in ${ }^{18} \mathrm{~F}$-alfatide II uptake $(P>0.05)$ (Table 6). In comparison, ${ }^{18} \mathrm{~F}$-FDG uptake of HER-2-overexpressing

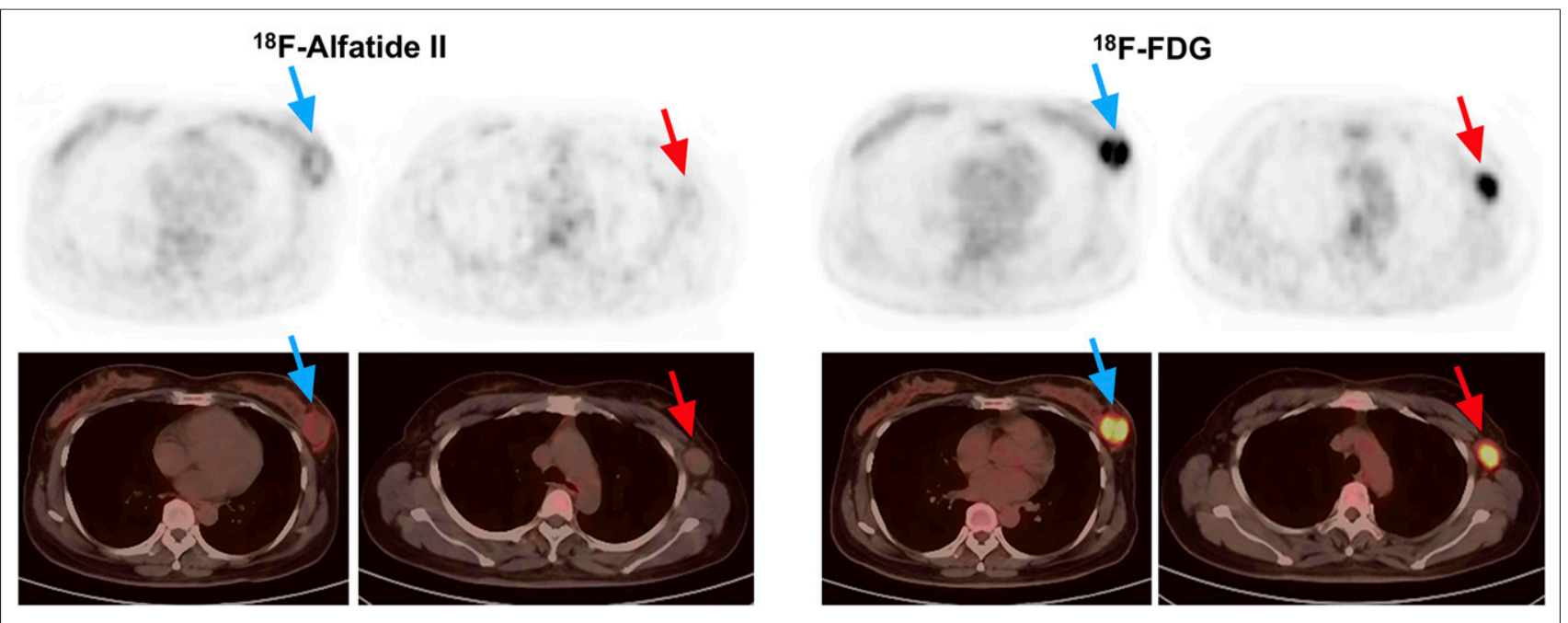

FIGURE 4. A 46-y-old patient with triple-negative breast cancer (blue arrows) and axillary lymph node metastasis (red arrows) showing no increased ${ }^{18} \mathrm{~F}$-alfatide II uptake but intense ${ }^{18} \mathrm{~F}-\mathrm{FDG}$ uptake. 
TABLE 4

Comparisons of ${ }^{18} \mathrm{~F}$-Alfatide II and ${ }^{18} \mathrm{~F}$-FDG Uptake in Breast Cancer and Benign Breast Lesion

\begin{tabular}{|c|c|c|c|c|c|}
\hline \multirow[b]{2}{*}{ Tracer } & & \multicolumn{2}{|c|}{$\mathrm{BC}$} & \multicolumn{2}{|c|}{$\mathrm{BBL}$} \\
\hline & & SUV $_{\max }$ & $S_{S U}$ mean & SUV $_{\max }$ & $S U V_{\text {mean }}$ \\
\hline \multirow[t]{2}{*}{${ }^{18} \mathrm{~F}$-alfatide II } & & $3.77 \pm 1.78$ & $2.25 \pm 0.98$ & $2.37 \pm 1.62$ & $1.50 \pm 0.92$ \\
\hline & $P$ value & $P<0.05$ & $P<0.05$ & $P>0.05$ & $P>0.05$ \\
\hline${ }^{18} \mathrm{~F}-\mathrm{FDG}$ & & $7.37 \pm 4.48$ & $4.54 \pm 2.82$ & $2.88 \pm 2.77$ & $1.75 \pm 1.50$ \\
\hline
\end{tabular}

subtypes was significantly higher than that of the other 3 subtypes $(P<0.05)$. Moreover, ${ }^{18} \mathrm{~F}$-FDG uptake in triple-negative subtype was significantly higher than that in luminal B subtype $(P<0.05)$. There was no significant difference in ${ }^{18} \mathrm{~F}-\mathrm{FDG}$ uptake between luminal A subtype and luminal B subtype, or between luminal A subtype and triple-negative subtype $(P>$ 0.05) (Table 7).

Eight breast cancer lesions had higher ${ }^{18} \mathrm{~F}$-alfatide II uptake than ${ }^{18}$ F-FDG. Among them, 6 lesions were luminal B subtype (HER-2-negative), which accounted for $66.7 \%$ (6/9) in all cases of this subtype (Fig. 6A). The remaining 2 lesions were luminal A subtype, with a proportion of $33.3 \%(2 / 6)$ of this subtype (Fig. $6 \mathrm{~B})$. Moreover, negative HER-2 expression and strongly positive (3+) ER expression were together shown in the 8 breast cancer lesions. The PR expression was diverse in these lesions.

\section{DISCUSSION}

RGD-based PET tracers have undergone rapid development in recent years. ${ }^{18} \mathrm{~F}$-galacto-RGD was the first noninvasive probe to target integrin $\alpha_{\mathrm{v}} \beta_{3}$ for PET angiogenesis imaging. Like ${ }^{18} \mathrm{~F}-$ Galacto-RGD, ${ }^{18} \mathrm{~F}$-AH111585 is another monomeric RGD PET tracer. There have been several preclinical and clinical studies suggesting the usefulness of these 2 PET tracers (20,22,26-30). However, they have relatively low tumor uptake, because of limited RGD binding of monomeric peptides and rapid clearance of the peptide tracers. Accordingly, radionuclide-labeled RGD dimers, including ${ }^{18} \mathrm{~F}-\mathrm{FPPRGD} 2$ and ${ }^{68} \mathrm{Ga}-\mathrm{PRGD} 2$, have been further developed to achieve better performance (31-33). The use of a fluoride-aluminum complex, ${ }^{18} \mathrm{~F}$-Al-NOTA-PRGD2 (denoted as ${ }^{18} \mathrm{~F}$-alfatide), significantly simplifies the labeling procedure (34-37).

For the first time, ${ }^{18} \mathrm{~F}$-alfatide II was clinically used in differentiating breast cancer from benign breast lesions in our study. As compared with previous studies using other RGD-based PET tracers in breast cancer patients $(19,20,22-24)$, our study included more diverse cases, such as breast fibroadenoma, breast adenosis, DCIS, invasive carcinoma, and lobular carcinoma. Even though there were 5 cases of early cancer in our study, breast cancer overall still showed relatively high ${ }^{18} \mathrm{~F}$-alfatide II uptake with a $\mathrm{SUV}_{\text {max }}$ of $3.77 \pm 1.78$ and $\mathrm{SUV}_{\text {mean }}$ of $2.25 \pm 0.98$, higher than that in benign breast lesions, which demonstrated that ${ }^{18} \mathrm{~F}$-alfatide II is suitable for identification of breast cancer in clinical practice.

In comparison with ${ }^{18} \mathrm{~F}-\mathrm{FDG},{ }^{18} \mathrm{~F}$-alfatide II had less difference in uptake between breast cancer and benign lesions. AUC of ${ }^{18} \mathrm{~F}$ alfatide II was also lower than that of ${ }^{18} \mathrm{~F}$ FDG in diagnosis of breast cancer. Some diagnostic parameters such as Youden index $\left(\mathrm{SUV}_{\max }\right)$, sensitivity, PPV, and NPV for ${ }^{18} \mathrm{~F}$-alfatide II were slightly lower than those for ${ }^{18} \mathrm{~F}-\mathrm{FDG}$, whereas the specificity was same. These results indicated that ${ }^{18} \mathrm{~F}$ alfatide II has a diagnostic value comparable to that of ${ }^{18} \mathrm{~F}$-FDG but is not superior in identification of breast cancer.

In our study, 5 cases of benign breast lesions displayed false-positive uptake for both ${ }^{18} \mathrm{~F}$-alfatide II and ${ }^{18} \mathrm{~F}$-FDG, suggesting that ${ }^{18} \mathrm{~F}$-alfatide II does not help improve the detection specificity. As for lobular carcinoma, it has been known that the false-negative rate of ${ }^{18} \mathrm{~F}-\mathrm{FDG}$ is as high as $65.2 \%$ (38). One case of lobular carcinoma included in our investigation also showed no ${ }^{18} \mathrm{~F}$-FDG uptake but had intense ${ }^{18} \mathrm{~F}$-alfatide II uptake (Fig. 4B). This phenomenon was consistent with that reported in previous studies using ${ }^{18} \mathrm{~F}$ FPPRGD2 (19), indicating that RGDbased PET tracers such as ${ }^{18} \mathrm{~F}$-alfatide II can be complementary to ${ }^{18} \mathrm{~F}-\mathrm{FDG}$ in diagnosis of lobular carcinoma of the breast.
FIGURE 5. Correlation between ${ }^{18} \mathrm{~F}$-alfatide II uptake and ${ }^{18} \mathrm{~F}-\mathrm{FDG}$ uptake, respectively, based on SUV $\mathrm{max}_{\text {max }}$ and SUV $\mathrm{V}_{\text {mean }}$ in breast cancer (A and B) and benign breast lesions (C and D). 
TABLE 5

${ }^{18} \mathrm{~F}-$ Alfatide II and ${ }^{18} \mathrm{~F}-\mathrm{FDG}$ Uptake in Different Molecular Subtypes of Breast Cancer

\begin{tabular}{|c|c|c|c|c|}
\hline \multirow[b]{2}{*}{ Molecular subtype } & \multicolumn{2}{|c|}{${ }^{18} \mathrm{~F}$-alfatide II } & \multicolumn{2}{|c|}{${ }^{18} \mathrm{~F}-\mathrm{FDG}$} \\
\hline & $S_{\text {SUax }}$ & $\mathrm{SUV}_{\text {mean }}$ & $\mathrm{SUV}_{\max }$ & $\mathrm{SUV}_{\text {mean }}$ \\
\hline Luminal A & $4.72 \pm 2.56$ & $2.87 \pm 1.42$ & $6.08 \pm 3.75$ & $3.77 \pm 2.30$ \\
\hline Luminal B & $4.35 \pm 1.72$ & $2.62 \pm 1.10$ & $5.36 \pm 2.64$ & $3.22 \pm 1.63$ \\
\hline HER-2 overexpressing & $4.41 \pm 1.92$ & $2.61 \pm 1.03$ & $13.88 \pm 3.89$ & $8.61 \pm 2.23$ \\
\hline Triple negative & $2.83 \pm 0.82$ & $1.77 \pm 0.53$ & $9.30 \pm 4.11$ & $5.80 \pm 2.69$ \\
\hline
\end{tabular}

TABLE 6

$P$ Values of Multiple Comparisons Using ${ }^{18} \mathrm{~F}$-Alfatide II SUV $\max$ Among Different Molecular Subtypes of Breast Cancer

\begin{tabular}{lcccc}
\hline \multicolumn{1}{c}{ Molecular subtype } & Luminal A & Luminal B & HER-2 positive & Triple negative \\
\hline Luminal A & - & 0.658 & 0.763 & 0.032 \\
Luminal B & 0.658 & - & 0.951 & 0.026 \\
HER-2 overexpressing & 0.763 & 0.951 & - & 0.088 \\
Triple negative & 0.032 & 0.026 & 0.088 & - \\
\hline
\end{tabular}

TABLE 7

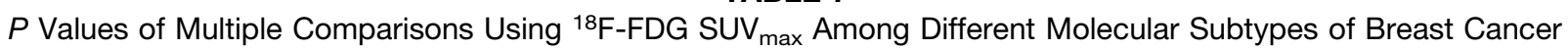

\begin{tabular}{lcccc}
\hline \multicolumn{1}{c}{ Molecular subtype } & Luminal A & Luminal B & HER-2 positive & Triple negative \\
\hline Luminal A & - & 0.67 & 0.001 & 0.074 \\
Luminal B & 0.670 & - & 0.000 & 0.006 \\
HER-2 overexpressing & 0.001 & 0.000 & - & 0.019 \\
Triple negative & 0.074 & 0.006 & 0.019 & - \\
\hline
\end{tabular}

Other false-negative cases of ${ }^{18} \mathrm{~F}-\mathrm{FDG}$ included 1 case of DCIS and 2 cases of breast cancer with luminal B subtype (negative HER-2 expression). The former case was also deficient in ${ }^{18} \mathrm{~F}-$ alfatide II uptake because of the small lesion size. The latter 2 cases, however, showed increased ${ }^{18} \mathrm{~F}$-alfatide II uptake, suggesting the additive role of ${ }^{18} \mathrm{~F}$-alfatide II to ${ }^{18} \mathrm{~F}-\mathrm{FDG}$ in detecting this subtype of breast cancer. On the other hand, 4 cases of triple-negative subtype without ${ }^{18} \mathrm{~F}$-alfatide II foci showed significantly increased ${ }^{18}$ F-FDG uptake, demonstrating that ${ }^{18} \mathrm{~F}-\mathrm{FDG}$ can compensate for the deficiency of ${ }^{18} \mathrm{~F}$-alfatide $\mathrm{II}$ in this subtype. Accordingly, combining ${ }^{18} \mathrm{~F}-$ alfatide II and ${ }^{18} \mathrm{~F}$-FDG together can significantly improve the sensitivity and NPV but may not add much in terms of the specificity and PPV.

Comparisons between ${ }^{18} \mathrm{~F}$-alfatide II and ${ }^{18} \mathrm{~F}$-FDG uptake were also made in our study. The result showed that tumor uptake of ${ }^{18} \mathrm{~F}$-alfatide II was significantly lower than that of ${ }^{18} \mathrm{~F}$ FDG in breast cancer. This finding was similar to those presented in other RGD tracer studies $(19,21)$. It has been reported in several studies that there was no significant correlation between RGD-based PET tracers and ${ }^{18}$ F-FDG in cancer lesion uptake $(21,24)$. Likewise, our result revealed a lack of significant correlation between ${ }^{18} \mathrm{~F}$-alfatide II uptake and ${ }^{18} \mathrm{~F}$-FDG uptake in breast cancer.

${ }^{18} \mathrm{~F}$-alfatide II uptake in different molecular subtypes of breast cancer was also assessed in our study. The triple-negative subtype showed no or low ${ }^{18} \mathrm{~F}$-alfatide II uptake, which was significantly lower than that of luminal A and luminal B subtypes. By contrast, the triple-negative subtype showed high ${ }^{18} \mathrm{~F}-\mathrm{FDG}$ uptake, whereas luminal A and luminal B subtypes had relatively low ${ }^{18} \mathrm{~F}$-FDG uptake. This result was similar to that from the previous study using ${ }^{68} \mathrm{Ga}-\mathrm{PRGD} 2$ in breast cancer patients (23).

Furthermore, our study evaluated the breast cancer lesions with higher uptake of RGD peptide than that of ${ }^{18} \mathrm{~F}-\mathrm{FDG}$ for the first time. A total of 8 such lesions were found in our study. Interestingly, they shared a common feature of being HER-2-negative and strongly ER-positive $(3+)$. This finding may help guide the therapeutic direction of breast cancer patients with multiple metastases, which are not classified by conventional molecular subtypes. If these metastases have higher uptake of ${ }^{18} \mathrm{~F}$-alfatide II than that of ${ }^{18} \mathrm{~F}$-FDG, they are very likely to be strongly ER-positive and HER-2-negative. Accordingly, they could benefit from endocrine therapy such as tamoxifen, but not from anti-HER-2 therapy such as trastuzumab.

There exist some limitations in our study. First, the number of participants is not large enough. Second, lymph node and other distant metastases were not evaluated due to the limited number of metastatic lesions in these patients. Third, immunohistochemistry tests were not performed to assess the correlation between integrin $\alpha_{v} \beta_{3}$ expression and alfatide II uptake, which 


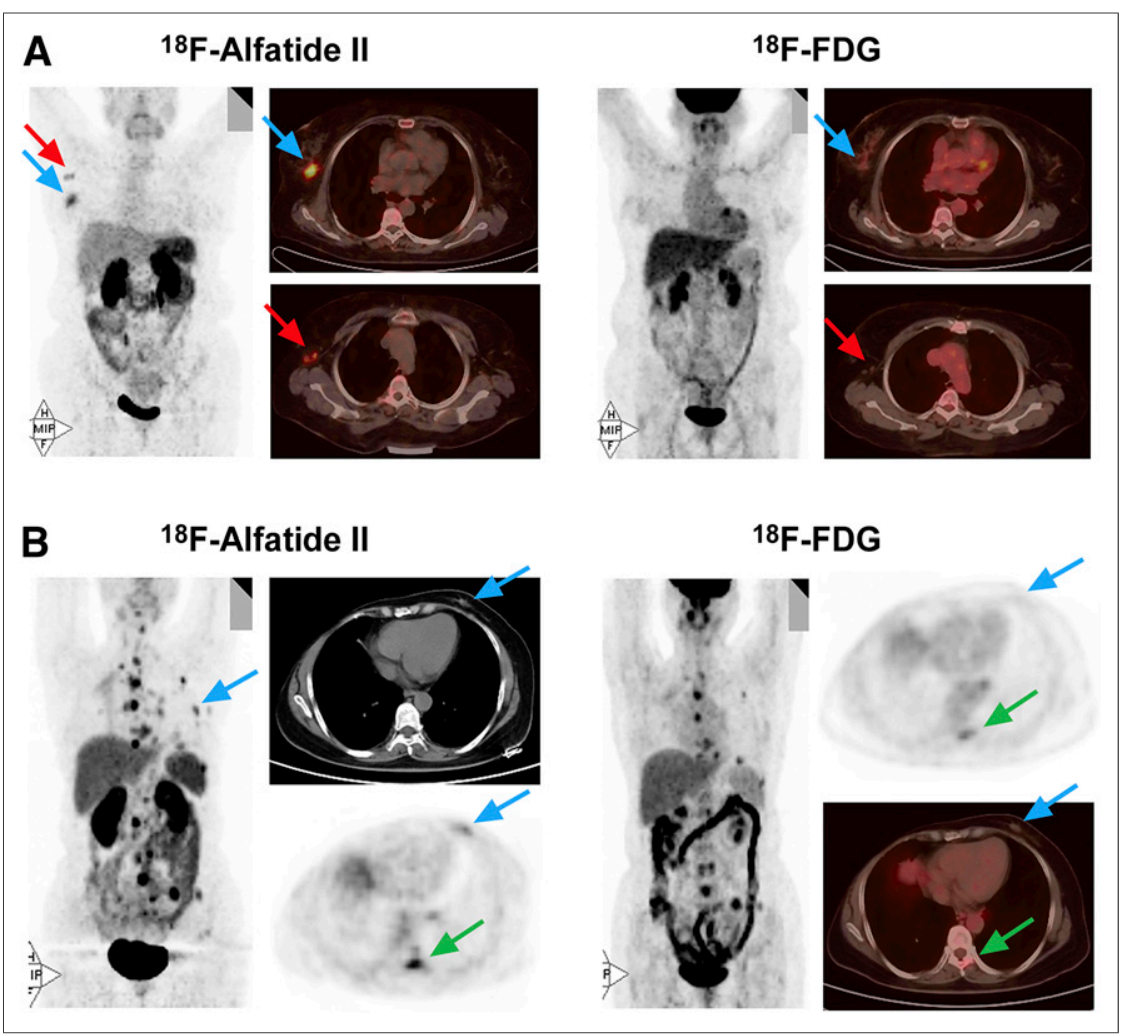

FIGURE 6. (A) A 60-y-old patient with luminal B (HER-2-negative) breast cancer (blue arrows) and axillary lymph node metastasis (red arrows) showing intense ${ }^{18} \mathrm{~F}$-alfatide II uptake but no ${ }^{18} \mathrm{~F}$ FDG uptake. (B) A 54-y-old patient with luminal A (HER-2-negative) breast cancer (blue arrows) showing increased ${ }^{18} \mathrm{~F}$-alfatide II uptake, but no ${ }^{18} \mathrm{~F}$-FDG uptake, and distant metastases with larger numbers and more intense uptake (green arrows) by ${ }^{18} \mathrm{~F}$-alfatide II than by ${ }^{18} \mathrm{~F}$-FDG.

\section{REFERENCES}

1. Brooks PC, Clark RA, Cheresh DA. Requirement of vascular integrin alpha $\mathrm{v}$ beta 3 for angiogenesis. Science. 1994;264:569-571.

2. Felding-Habermann B, O'Toole TE, Smith JW, et al. Integrin activation controls metastasis in human breast cancer. Proc Natl Acad Sci USA. 2001;98:1853-1858.

3. Hood JD, Cheresh DA. Role of integrins in cell invasion and migration. Nat Rev Cancer. 2002; 2:91-100.

4. Danhier F, Le Breton A, Preat V. RGD-based strategies to target $\alpha v \beta 3$ integrin in cancer therapy and diagnosis. Mol Pharm. 2012;9:29612973.

5. Chen H, Niu G, Wu H, et al. Clinical application of radiolabeled RGD peptides for PET imaging of integrin alphavbeta3. Theranostics. 2016;6: 78-92.

6. Chakravarty R, Chakraborty S, Dash A. Molecular imaging of breast cancer: role of RGD peptides. Mini Rev Med Chem. 2015;15:1073-1094.

7. Chen X, Park R, Hou Y, et al. MicroPET imaging of brain tumor angiogenesis with 18F-labeled PEGylated RGD peptide. Eur J Nucl Med Mol Imaging. 2004;31:1081-1089.

8. Jackson $\mathrm{AB}$, Nanda $\mathrm{PK}$, Rold TL, et al. ${ }^{64} \mathrm{Cu}-$ NO2A-RGD-Glu-6-Ahx-BBN(7-14)NH2: a heterodimeric targeting vector for positron emission tomography imaging of prostate cancer. $\mathrm{Nucl} \mathrm{Med}$ Biol. 2012;39:377-387.

9. Eo JS, Jeong JM. Angiogenesis imaging using ${ }^{68} \mathrm{Ga}$-RGD PET/CT: therapeutic implications. Semin Nucl Med. 2016;46:419-427.

10. Jacobson O, Zhu L, Niu G, et al. MicroPET imaging of integrin $\alpha \mathrm{v} \beta 3$ expressing tumors using ${ }^{89} \mathrm{Zr}-\mathrm{RGD}$ peptides. Mol Imaging Biol. 2011; 13:1224-1233.

11. Guo J, Guo N, Lang L, et al. ${ }^{18} \mathrm{~F}$-alfatide II and has been demonstrated in several animal and clinical studies $(12,16,39)$. Further investigations are still required in the future to elucidate the role of ${ }^{18} \mathrm{~F}$-alfatide II in breast cancer management.

\section{CONCLUSION}

${ }^{18} \mathrm{~F}$-alfatide II is clinically amenable for the identification of breast cancer without special patient preparation. ${ }^{18} \mathrm{~F}$-alfatide II has good diagnostic value in distinguishing between breast cancer and benign breast lesions, but is not superior to ${ }^{18} \mathrm{~F}-\mathrm{FDG} .{ }^{18} \mathrm{~F}-$ alfatide II can exert a complementary role to ${ }^{18} \mathrm{~F}-\mathrm{FDG}$ in breast lobular carcinoma and other breast cancers with strongly positive ER expression and negative HER-2 expression.

\section{DISCLOSURE}

This work was supported by the National Key Basic Research Program of China (973 program, 2014CB744504), the National Natural Science Foundation of China (81501537), the Natural Science Foundation of Jiangsu Province (BK20160610), Jiangsu Province Social Development Program (BE2017772), Jiangsu Planned Projects for Postdoctoral Research Funds (1601090C), and the Intramural Research Program, National Institute of Biomedical Imaging and Bioengineering, National Institutes of Health. Jihong Tian, Chuanjin Sun, and Xingang Wang also contributed to this work. No other potential conflict of interest relevant to this article was reported.
${ }^{18} \mathrm{~F}-\mathrm{FDG}$ dual-tracer dynamic PET for parametric, early prediction of tumor response to therapy. J Nucl Med. 2014;55:154-160.

12. Wu C, Yue X, Lang L, et al. Longitudinal PET imaging of muscular inflammation using ${ }^{18} \mathrm{~F}$-DPA-714 and ${ }^{18} \mathrm{~F}$-alfatide II and differentiation with tumors. Theranostics. 2014;4:546-555.

13. Wang SY, Bao X, Wang MW, et al. Radiation dosimetry estimates of ${ }^{18} \mathrm{~F}$-alfatide II based on whole-body PET imaging of mice. Appl Radiat Isot. 2015;105:1-5.

14. Yu C, Pan D, Mi B, et al. ${ }^{18} \mathrm{~F}$-alfatide II PET/CT in healthy human volunteers and patients with brain metastases. Eur J Nucl Med Mol Imaging. 2015;42:20212028.

15. Mi B, Yu C, Pan D, et al. Pilot prospective evaluation of ${ }^{18} \mathrm{~F}$-alfatide II for detection of skeletal metastases. Theranostics. 2015;5:1115-1121.

16. Bao X, Wang MW, Luo JM, et al. Optimization of early response monitoring and prediction of cancer antiangiogenesis therapy via noninvasive PET molecular imaging strategies of multifactorial bioparameters. Theranostics. 2016;6:2084-2098.

17. Mackey JR, Kerbel RS, Gelmon KA, et al. Controlling angiogenesis in breast cancer: a systematic review of anti-angiogenic trials. Cancer Treat Rev. 2012;38:673-688.

18. Lopes G, Dent R. Weighed, measured, and still searching: bevacizumab in the treatment of unselected patients with advanced breast cancer. Oncologist. 2011;16:1669-1671.

19. Iagaru A, Mosci C, Shen B, et al. ${ }^{18}$ F-FPPRGD2 PET/CT: pilot phase evaluation of breast cancer patients. Radiology. 2014;273:549-559.

20. Beer AJ, Niemeyer M, Carlsen J, et al. Patterns of $\alpha_{v} \beta_{3}$ expression in primary and metastatic human breast cancer as shown by ${ }^{18} \mathrm{~F}$-galacto-RGD PET. J Nucl Med. 2008;49:255-259.

21. Beer AJ, Lorenzen S, Metz S, et al. Comparison of integrin $\alpha_{\mathrm{v}} \beta_{3}$ expression and glucose metabolism in primary and metastatic lesions in cancer patients: a PET study using ${ }^{18}$ F-galacto-RGD and ${ }^{18}$ F-FDG. J Nucl Med. 2008;49:22-29.

22. Kenny LM, Coombes RC, Oulie I, et al. Phase I trial of the positron-emitting Arg-Gly-Asp (RGD) peptide radioligand ${ }^{18} \mathrm{~F}-\mathrm{AH} 111585$ in breast cancer patients. J Nucl Med. 2008;49:879-886. 
23. Yoon HJ, Kang KW, Chun IK, et al. Correlation of breast cancer subtypes, based on estrogen receptor, progesterone receptor, and HER2, with functional imaging parameters from ${ }^{68} \mathrm{Ga}-\mathrm{RGD}$ PET/CT and ${ }^{18} \mathrm{~F}-\mathrm{FDG}$ PET/CT. Eur J Nucl Med Mol Imaging. 2014;41:1534-1543.

24. Minamimoto R, Jamali M, Barkhodari A, et al. Biodistribution of the ${ }^{18} \mathrm{~F}$ FPPRGD $_{2}$ PET radiopharmaceutical in cancer patients: an atlas of SUV measurements. Eur J Nucl Med Mol Imaging. 2015;42:1850-1858.

25. Lang L, Ma Y, Kiesewetter DO, et al. Stability analysis of glutamic acid linked peptides coupled to NOTA through different chemical linkages. Mol Pharm. 2014;11:3867-3874.

26. Beer AJ, Haubner R, Goebel M, et al. Biodistribution and pharmacokinetics of the $\alpha_{\mathrm{v}} \beta_{3}$-selective tracer ${ }^{18} \mathrm{~F}$-galacto-RGD in cancer patients. J Nucl Med. 2005;46:1333-1341.

27. Beer AJ, Haubner R, Wolf I, et al. PET-based human dosimetry of ${ }^{18} \mathrm{~F}$-galacto-RGD, a new radiotracer for imaging $\alpha_{\mathrm{v}} \beta_{3}$ expression. J Nucl Med. 2006;47:763-769.

28. Beer AJ, Grosu AL, Carlsen J, et al. $\left[{ }^{18} \mathrm{~F}\right]$ galacto-RGD positron emission tomography for imaging of alphavbeta3 expression on the neovasculature in patients with squamous cell carcinoma of the head and neck. Clin Cancer Res. 2007;13: 6610-6616.

29. Beer AJ, Haubner R, Sarbia M, et al. Positron emission tomography using $\left[{ }^{18} \mathrm{~F}\right]$ galacto-RGD identifies the level of integrin $\alpha \mathrm{v} \beta 3$ expression in man. Clin Cancer Res. 2006;12:3942-3949.

30. McParland BJ, Miller MP, Spinks TJ, et al. The biodistribution and radiation dosimetry of the Arg-Gly-Asp peptide ${ }^{18} \mathrm{~F}$-AH111585 in healthy volunteers. $J$ Nucl Med. 2008;49:1664-1667.
31. Li ZB, Chen K, Chen X. ${ }^{68}$ Ga-labeled multimeric RGD peptides for microPET imaging of integrin $\alpha v \beta 3$ expression. Eur J Nucl Med Mol Imaging. 2008;35:1100-1108.

32. Liu S, Liu Z, Chen K, et al. ${ }^{18}$ F-labeled galacto and PEGylated RGD dimers for PET imaging of $\alpha \mathrm{v} \beta 3$ integrin expression. Mol Imaging Biol. 2010;12:530538.

33. Mittra ES, Goris ML, Iagaru AH, et al. Pilot pharmacokinetic and dosimetric studies of ${ }^{18} \mathrm{~F}$-FPPRGD2: a PET radiopharmaceutical agent for imaging $\alpha \mathrm{v} \beta 3$ integrin levels. Radiology. 2011;260:182-191.

34. Liu S, Liu H, Jiang H, et al. One-step radiosynthesis of ${ }^{18} \mathrm{~F}-A$ IF-NOTA-RGD2 for tumor angiogenesis PET imaging. Eur J Nucl Med Mol Imaging. 2011;38: $1732-1741$.

35. Wan W, Guo N, Pan D, et al. First experience of ${ }^{18} \mathrm{~F}$-alfatide in lung cancer patients using a new lyophilized kit for rapid radiofluorination. J Nucl Med. 2013;54:691-698.

36. Zhou Y, Gao S, Huang Y, et al. A pilot study of ${ }^{18} \mathrm{~F}$-Alfatide PET/CT imaging for detecting lymph node metastases in patients with non-small cell lung cancer. Sci Rep. 2017;7:2877.

37. Guo J, Lang L, Hu S, et al. Comparison of three dimeric ${ }^{18} \mathrm{~F}-\mathrm{AlF}-\mathrm{NOTA}-\mathrm{RGD}$ tracers. Mol Imaging Biol. 2014;16:274-283.

38. Bombardieri E, Aktolun C, Baum RP, et al. FDG-PET: procedure guidelines for tumour imaging. Eur J Nucl Med Mol Imaging. 2003;30:BP115-BP124.

39. Kang F, Wang S, Tian F, et al. Comparing the diagnostic potential of ${ }^{68} \mathrm{Ga}-$ alfatide II and ${ }^{18} \mathrm{~F}-\mathrm{FDG}$ in differentiating between non-small cell lung cancer and tuberculosis. J Nucl Med. 2016;57:672-677. 